\title{
Variability of Paranasal Sinus Pneumatization in the Absence of Sinus Disease
}

\author{
Michael J. Marino, MD, ${ }^{1}$ Charles A. Riley, MD, ${ }^{2}$ Eric L. Wu, MD, ${ }^{3}$ Jacqueline E. Weinstein, MD, ${ }^{4}$ Noah Emerson, DO, ${ }^{5}$ \\ Edward D. McCoul, MD, MPH ${ }^{3,6,7}$

\begin{abstract}
${ }^{1}$ Department of Otorhinolaryngology, Mayo Clinic, Phoenix, AZ 2 Division of Otolaryngology, Fort Belvoir Community Hospital, Fort Belvoir, VA ${ }^{3}$ Department of Otolaryngology-Head and Neck Surgery, Tulane University School of Medicine, New Orleans, LA ${ }^{4}$ Division of Pediatric Otolaryngology-Head and Neck Surgery, Benioff Children's Hospital, University of California, San Francisco, San Francisco, CA

${ }^{5}$ Department of Radiology, Ochsner Clinic Foundation, New Orleans, LA ${ }^{6}$ Department of Otorhinolaryngology, Ochsner Clinic Foundation, New Orleans, LA ${ }^{7}$ The University of Queensland Faculty of Medicine, Ochsner Clinical School, New Orleans, LA
\end{abstract}

Background: Paranasal sinus pneumatization is a complex process, and numerous computed tomography (CT) studies document developmental variations in the setting of underlying sinus disease. The purpose of this study was to investigate variation in paranasal sinus pneumatization in a population of nondiseased subjects using a metric validated for tracking individual anatomic variants as well as total sinus volume.

Methods: A total of 591 consecutive sinus and maxillofacial CT scans were considered for study inclusion. After patients with inflammatory sinus or respiratory disease were excluded, 323 CT scans were scored using the Assessment of Pneumatization of the Paranasal Sinuses (APPS) instrument, and relevant demographic data were recorded for each scan. APPS findings were compared according to demographic characteristics and laterality.

Results: Laterality and sex were associated with differences in paranasal sinus pneumatization in a nondiseased population. Based on APPS score, the left side (4.95) was more pneumatized than the right $(4.74, P=0.006)$, and males (10.16) were more extensively pneumatized than females $(9.18, P=0.005)$. We found no correlation of age with sinus pneumatization $(\rho=0.025)$. The probability of perceptible asymmetry in any given individual's paranasal sinus pneumatization was $69 \%$, and the probability of left-sided dominance was $53 \%$.

Conclusion: Substantial anatomic variation exists in paranasal sinus anatomy, even among patients without sinus disease. Significant differences are found between males and females and between the left and right sides. Continued systematic research of paranasal sinus anatomy may facilitate a standard for CT sinus assessment that will aid clinician evaluation of anatomic variation and surgical decision making.

Keywords: Anatomic variation, paranasal sinuses, tomography- $x$-ray computed

Address correspondence to Edward D. McCoul, MD, MPH, Department of Otorhinolaryngology, Ochsner Clinic Foundation, 1514 Jefferson Hwy., New Orleans, LA 70121. Tel: (504) 842-4080. Email: emccoul@gmail.com

\section{INTRODUCTION}

Paranasal sinus pneumatization is a complex and incompletely understood process. Multiple reports associate the anatomic variations seen on sinus computed tomography (CT) with specific sinonasal diseases. Paranasal sinus hypoplasia has been well established in cystic fibrosis, ${ }^{1-4}$ and similar variations have been reported in patients with primary ciliary dyskinesia and Kartagener syndrome. ${ }^{5}$ Variations of paranasal sinus pneumatization in the setting of chronic rhinosinusitis have been less clear. Studies have reported decreased maxillary sinus pneumatization, ${ }^{6,7}$ increased frontal sinus pneumatization, ${ }^{8}$ or no differences compared to patients without chronic rhinosinusitis. ${ }^{9}$ Multiple theories have been proffered to account for the differences in paranasal sinus pneumatization, including the effect of chronic sinus inflammation and infection, 9,10 genetic mechanisms, ${ }^{1,3,4}$ regional blood flow anamolies, ${ }^{11,12}$ and increased serum erythropoietin. ${ }^{11}$

In addition to the potential associations between differential sinus pneumatization and clinical disease states, nondiseased patients also appear to have considerable anatomic variation. The presence of at least a single sinonasal anatomic variant has been reported to be $64.0 \%$ to $99.8 \%$ in studies of patients with and without mucosal sinus disease..$^{13-15}$ As many as 52 bony or air cell variants have been identified, and up to $41 \%$ were without apparent impact on clinical disease, endoscopic vision, or exposure of critical structures. ${ }^{15}$ Nevertheless, differing definitions of specific anatomic variations remain a concern for reliable identification. ${ }^{16}$ Defining the degree of paranasal 
Table 1. Assessment of Pneumatization of the Paranasal Sinuses Items

\begin{tabular}{ll}
\hline Item & \multicolumn{1}{c}{ Anatomic Variant } \\
\hline 1 & Maxillary floor inferior to nasal floor \\
2 & $\begin{array}{c}\text { Supraorbital cell (air cell superior to anterior } \\
\text { ethmoid artery) }\end{array}$ \\
3 & Middle turbinate concha bullosa present \\
4 & Frontal sinus present \\
5 & Superior frontal sinus wall superior to \\
6 & supraorbital rim \\
7 & Lateral frontal sinus wall lateral to medial edge \\
& of globe \\
9 & Lateral frontal sinus wall lateral to midpupillary \\
9 & Anterior clinoid process pneumatized
\end{tabular}

$\mathrm{V}_{2}$, maxillary nerve canal; $\mathrm{VN}$, Vidian nerve canal.

sinus anatomic variation and overall pneumatization using a validated method may be useful for clinicians in understanding baseline variability and could also help to identify intrinsic patient characteristics that are associated with differing pneumatization or specific variations.

The Assessment of Pneumatization of the Paranasal Sinuses (APPS) score was introduced as a radiographic instrument for tracking anatomic variation on sinus CT and is validated for interrater and intrarater reliability. ${ }^{17}$ The APPS instrument evaluates for the presence of 9 variants bilaterally (Table 1, Figure 1), and each item present is assigned a score of 1 for a total possible score range of 0 to 18 . The total APPS score has been shown to correlate strongly with the total sinus volume as calculated by 3-dimensional volumetric analysis of sinus CT. ${ }^{18}$

For this study, we used the APPS score as a validated metric to investigate the presence of individual anatomic sinus variants in a nondiseased population. Also, because the APPS score correlates with total sinus volume, we compared overall paranasal sinus pneumatization by demographic characteristics and laterality. Understanding anatomic and pneumatization variants may facilitate clinician evaluation of baseline paranasal sinus variation and identification of influencing factors.

\section{METHODS}

Approval for this study was obtained from the Ochsner Clinic Foundation Institutional Review Board. A total of 591 sinus and maxillofacial CT scans performed at the senior author's primary institution between January 1, 2010, and August 15, 2015, were evaluated for APPS and Lund-Mackay scores. The Lund-Mackay score is a validated instrument for measuring the degree of sinus opacification. ${ }^{17,19}$

Total APPS scores (ranging from 0 to 18) were used to assess overall paranasal sinus pneumatization, and laterality-based comparisons were performed using unilateral APPS scores (ranging from 0 to 9). Patients with inflammatory sinus or respiratory disease, including chronic
Table 2. Demographic and Radiographic Characteristics of the Study Population

\begin{tabular}{lc}
\hline \multicolumn{1}{c}{ Variable } & All Patients $(\mathbf{n = 3 2 3 )}$ \\
\hline Age, years, mean \pm SD & $32.6 \pm 22.9$ \\
Sex, $\mathrm{n}(\%)$ & \\
$\quad$ Male & $166(51.4)$ \\
$\quad$ Female & $157(48.6)$ \\
Ethnicity, ${ }^{\text {a }} \mathrm{n}(\%)$ & \\
Caucasian/white (non-Hispanic) & $201(62.2)$ \\
African American/black & $95(29.4)$ \\
Latinx/Hispanic & $18(5.6)$ \\
APPS score, mean \pm SD & $9.68 \pm 3.16$ \\
Lund-Mackay score, mean \pm SD & $2.21 \pm 2.50$ \\
\hline
\end{tabular}

${ }^{a}$ Nine patients reported a different ethnic group or declined to respond. APPS, Assessment of Pneumatization of the Paranasal Sinuses.

rhinosinusitis, allergic rhinitis, recurrent acute rhinosinusitis, cystic fibrosis, asthma, obstructive sleep apnea/sleepdisordered breathing, and maxillofacial fractures were excluded from the study. These diagnoses were ascertained retrospectively from the medical record. Also, only patients 13 years or older were included in this study, the age at which sinus pneumatization is presumed to be complete. ${ }^{20}$ A nondiseased population of 323 CT scans was included for analysis. The scans had been typically obtained for evaluation of suspected facial trauma, headache, or other nonsinonasal complaints. Radiographic scores and relevant demographic data, including age at the date of CT acquisition, ethnicity, and sex, were stored in a secure, web-based Research Electronic Data Capture v.6.6.2 (REDCap Vanderbilt University Medical Center) database for management and analysis.

The nondiseased population was analyzed for differences in paranasal sinus pneumatization according to demographic characteristics and laterality. The frequency of individual anatomic variants was also analyzed. Paired continuous variables were analyzed using paired $t$ tests, while unpaired data were compared using independent twosample $t$ tests. Analyses comparing more than 2 groups simultaneously were performed using one-way analysis of variance. Chi-square test was used to compare categorical variables. Correlation analysis was performed using the Spearman rho. Common language effect size index was used to determine effect size as a probability. ${ }^{21} P$ values $<0.05$ were considered significant. Statistical analysis was performed using SAS software v.9.3 (SAS Institute Inc.).

\section{RESULTS}

The demographic characteristics and mean radiographic scores of the entire study population are presented in Table 2. In the comparison of the extent of paranasal sinus pneumatization by sex, males had statistically significant increased pneumatization compared to females $(P=0.005$, Table 3). The anatomic variation among ethnic groups did not reach statistical significance $(P=0.148)$, and the extent of sinus pneumatization was not correlated with age $(\rho=0.025$, $P=0.657$, Figure 2). 

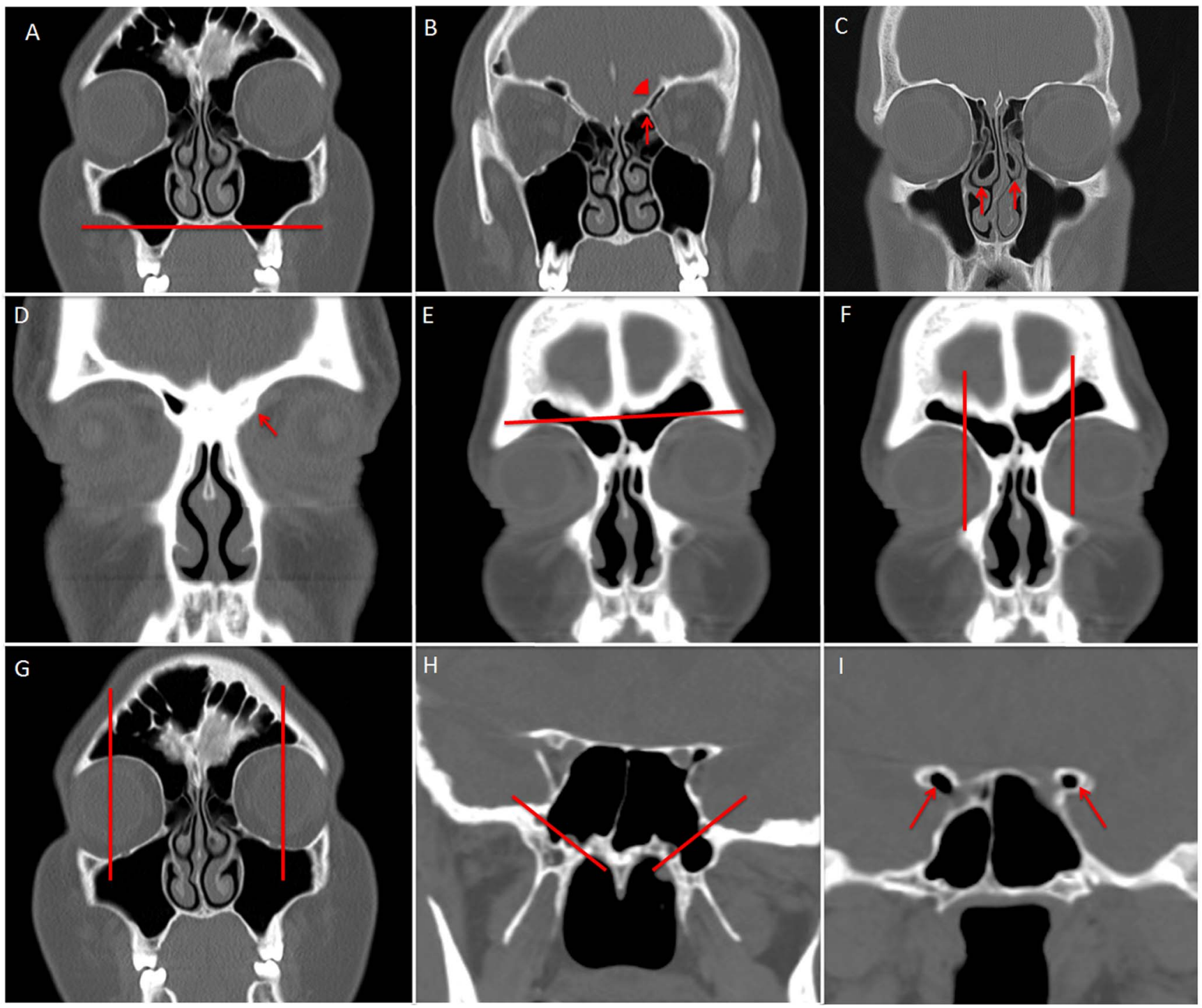

Figure 1. (A) The floor of the maxillary sinus extends inferior to the floor of the nasal cavity, corresponding with APPS item 1. (B) Supraorbital air cell (arrowhead) is present superior to the anterior ethmoid artery (arrow), corresponding with APPS item 2. (C) Middle turbinate concha bullosa is present (arrows), corresponding with APPS item 3. (D) The frontal sinus is absent (arrow), corresponding with APPS item 4. (E) The frontal sinus extends superior to the supraorbital rim, corresponding with APPS item 5. (F) The lateral wall of the frontal sinus extends lateral to the medial edge of the globe, corresponding with APPS item 6. (G) The lateral wall of the frontal sinus extends lateral to the midpupillary line, corresponding with APPS item 7. (H) The lateral wall of the sphenoid sinus extends beyond to the $\mathbf{V}_{\mathbf{2}}-\mathrm{VN}$ line, corresponding with APPS item 8 . (I) The anterior clinoid process is pneumatized (arrows), corresponding with APPS item 9. APPS, Assessment of Pneumatization of the Paranasal Sinuses; $\mathrm{V}_{2}$, maxillary nerve canal, VN, Vidian nerve canal.

In the comparison of paranasal sinus pneumatization according to laterality, the left side had statistically significant increased pneumatization compared to the right side

Table 3. Paranasal Sinus Pneumatization According to Sex

\begin{tabular}{lcc}
\hline & $\begin{array}{c}\text { Age, years, } \\
\text { mean } \pm \text { SD }\end{array}$ & $\begin{array}{c}\text { APPS Score, } \\
\text { mean } \pm \text { SD }\end{array}$ \\
\hline Male $(\mathrm{n}=166)$ & $27.7 \pm 19.3$ & $10.16 \pm 3.02$ \\
Female $(\mathrm{n}=157)$ & $37.7 \pm 25.3$ & $9.18 \pm 3.24$ \\
$P$ Value & $<0.001$ & 0.005
\end{tabular}

APPS, Assessment of Pneumatization of the Paranasal Sinuses.
$(P=0.006)$ (Table 4). This difference between sides increased when the more extensively pneumatized side (either left or right) was compared to the less pneumatized side $(P<0.001)$. The left side more frequently had increased pneumatization (36.5\% of subjects) vs the right side (30.7\% of subjects). Both sides were symmetric in $32.8 \%$ of cases. Asymmetry, in general, was more frequent than symmetry between the sides $(P<0.001)$. According to the common language effect size index, the probability of encountering asymmetry between sides in any given individual was $69 \%$, while the probability that the left side would be more highly pneumatized than the right was $53 \%$. The frequency of individual variations tracked in the APPS score are shown in Table 5. 


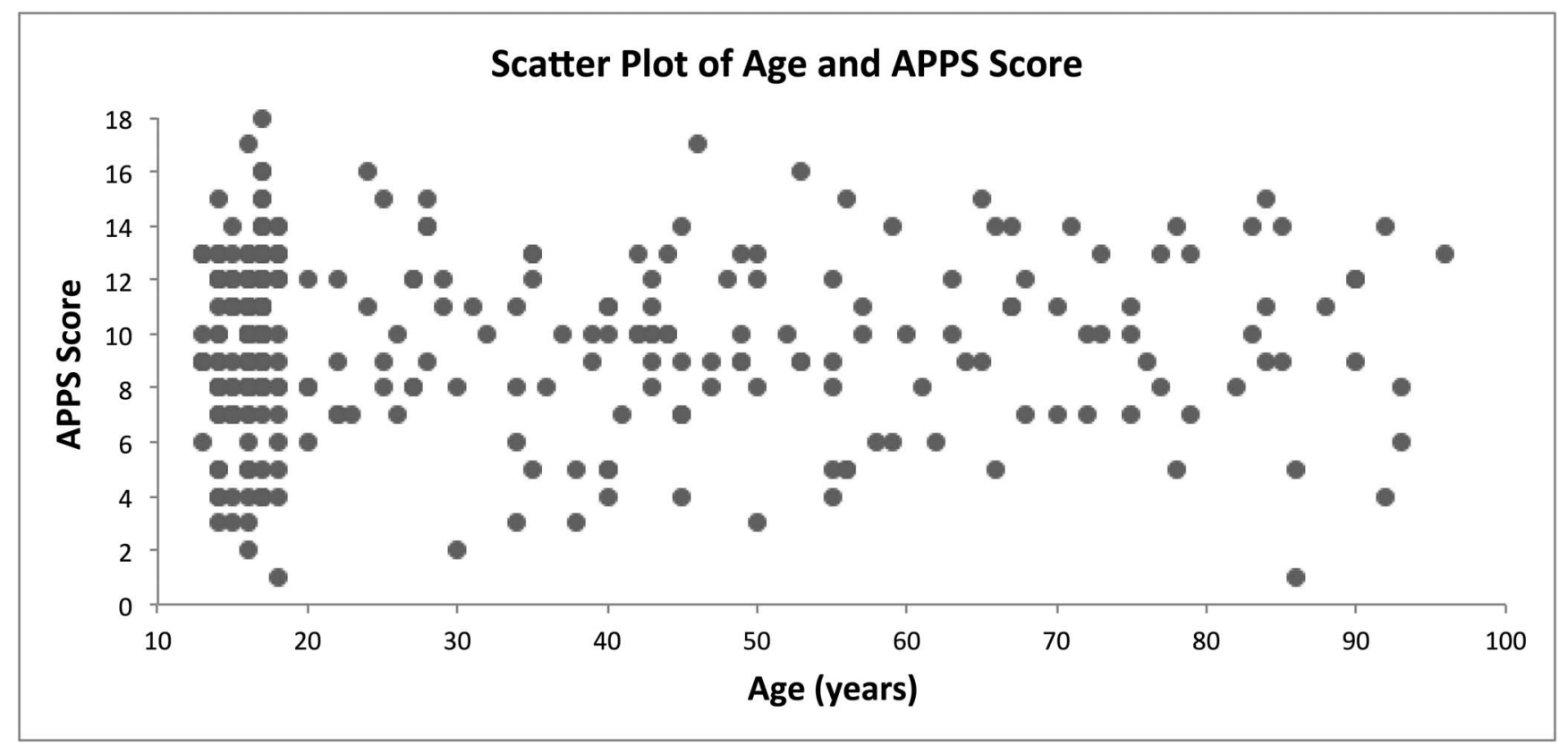

Figure 2. Scatter plot of age and Assessment of Pneumatization of the Paranasal Sinuses (APPS) score for all computed tomography scans included in the study $(n=323)$ shows no correlation between these parameters $(\rho=0.025, P=0.657)$.

\section{DISCUSSION}

Wide variation in paranasal sinus pneumatization appears to exist, and the mechanisms for these differences are poorly understood. While certain sinonasal or respiratory pathologies are associated with measurable changes in sinus pneumatization, these variations are not restricted to a diseased state. ${ }^{13-15}$ More than 50 variations of the bony and air cell structure of the paranasal sinuses have been described, and up to $40 \%$ may not correspond with disease presentation. ${ }^{15}$ Furthermore, pervasive differences in paranasal sinus pneumatization, according to parameters other than clinical disease, continue to be an area of investigation among anatomists and clinicians. ${ }^{22}$ Understanding the degree of variation in nondiseased patients might better contextualize aberrations seen in the setting of clinical disease and may also help in the development of standardized sinus CT reports to aid clinician interpretation of the relevant anatomy.

Global paranasal sinus pneumatization seems to differ between males and females, with male subjects having increased pneumatization (Table 3). The APPS score, used to estimate total sinus volume in this study, also tracks individual anatomic variations and showed a decreased number of

Table 4. Paranasal Sinus Pneumatization According to Laterality

\begin{tabular}{lcc}
\hline \multicolumn{1}{c}{ Side } & APPS Score, mean \pm SD & P Value \\
\hline Left & $4.95 \pm 1.77$ & 0.006 \\
Right & $4.74 \pm 1.68$ & \\
More pneumatized side & $5.35 \pm 1.64$ & $<0.001$ \\
Less pneumatized side & $4.33 \pm 1.66$ & \\
\hline
\end{tabular}

APPS, Assessment of Pneumatization of the Paranasal Sinuses. pneumatization variants in females. These findings are consistent with other reports of decreased volume of individual sinuses in females. ${ }^{23-26}$ Maxillary and frontal sinus volume on CT have even been reported to successfully differentiate between males and females in forensic analysis. ${ }^{24-26}$

In the current study, the male group was statistically younger than the female group, which may confound the finding of increased sinus pneumatization in males. We found no correlation, however, of age with the pneumatization of developed sinuses (Figure 2). As stated previously, all patients in this study were 13 years or older, at which point pneumatization was presumed to be complete. ${ }^{20}$ Reports of age-related pneumatization differences after complete sinus development conflict. ${ }^{23,27}$ Overall, our study suggests that among nondiseased patients, males have increased paranasal sinus pneumatization, and age-related differences are not present in completely developed sinuses.

Paranasal sinus morphology appears to remain stable across ethnic groups despite frequent variation among individuals. ${ }^{28}$ However, clinical sinus disease such as chronic rhinosinusitis remains an important health concern among different races and ethnicities. ${ }^{29}$ Investigations of anatomic differences according to ethnicity have been mixed. Some studies have demonstrated differences of particular variants and total sinus volume between specific ethnic groups, ${ }^{28,30}$ while others have found a similar degree of anatomic variation between ethnicities. ${ }^{31}$ Our study did not detect differences in overall paranasal sinus pneumatization between patients of Caucasian, African American, or Latinx/Hispanic ethnicity. Conclusions regarding ethnicity-related differences, however, are limited secondary to the small number of Latinx/Hispanic patients included in this study.

Comparisons of overall paranasal sinus pneumatization according to laterality have not been reported previously. In our study, increased pneumatization on the left side reached 
Table 5. Frequency of Individual Paranasal Sinus Anatomic Variations

\begin{tabular}{|c|c|c|c|c|}
\hline Item/Anatomic Variant & $\begin{array}{c}\text { Left } \\
\text { Prevalence } \\
(n=323)\end{array}$ & $\begin{array}{c}\text { Right } \\
\text { Prevalence } \\
(n=323)\end{array}$ & $\begin{array}{c}\text { Total } \\
\text { Prevalence } \\
(n=646)\end{array}$ & $\begin{array}{c}\text { PValue, } \\
\text { Right vs } \\
\text { Left }\end{array}$ \\
\hline 1. Maxillary floor inferior to nasal floor & $263(81.4)$ & $267(82.7)$ & $530(82.0)$ & 0.680 \\
\hline $\begin{array}{l}\text { 2. Supraorbital cell (air cell superior to anterior ethmoid } \\
\text { artery) }\end{array}$ & $80(24.8)$ & $76(23.5)$ & $156(24.1)$ & 0.777 \\
\hline 3. Middle turbinate concha bullosa present & $98(30.3)$ & $89(27.6)$ & $187(28.9)$ & 0.488 \\
\hline 4. Frontal sinus present & $310(96.0)$ & $307(95.0)$ & $617(95.5)$ & 0.572 \\
\hline 5. Superior frontal sinus wall superior to supraorbital rim & $282(87.3)$ & $274(84.8)$ & $556(86.1)$ & 0.362 \\
\hline 6. Lateral frontal sinus wall lateral to medial edge of globe & $259(80.2)$ & $254(78.6)$ & $513(79.4)$ & 0.624 \\
\hline 7. Lateral frontal sinus wall lateral to midpupillary line & $85(26.3)$ & $61(18.9)$ & $146(22.6)$ & 0.024 \\
\hline 8. Lateral sphenoid sinus wall lateral to $\mathrm{V}_{2}-\mathrm{VN}$ line & $172(53.3)$ & $145(44.9)$ & $317(49.1)$ & 0.034 \\
\hline 9. Anterior clinoid process pneumatized & $49(15.2)$ & $57(17.6)$ & $106(16.4)$ & 0.396 \\
\hline
\end{tabular}

Note: Data are presented as $\mathrm{n}(\%)$.

$\mathrm{V}_{2}$, maxillary nerve canal; $\mathrm{VN}$, Vidian nerve canal.

statistical significance (Table 4). Analysis of the individual variations tracked in the APPS score indicated that pneumatization lateral to a line drawn from the maxillary nerve canal to the Vidian nerve canal and frontal sinus pneumatization lateral to the midpupillary line were more frequent on the left (Table 5). Although statistically significant, the mean difference in APPS score between the right and left sides was small at 0.21 and may not have clinical significance. Alternatively, this difference could indicate selective pressures that influence laterality-based development in the head and neck. Embryologic mechanisms for laterality differences of anatomic structures in the head and neck, such as the course of recurrent laryngeal nerve and thoracic duct, are known. Similar mechanisms for sinonasal structures have been incompletely studied. Regardless of the specific side, the number of increased pneumatization variants tended to track together. The number of variants on the larger side was statistically greater than those on the smaller side, and the 2 sides were more frequently asymmetric. In $69 \%$ of cases, the paranasal sinus pneumatization would be expected to exhibit perceptible asymmetry. Moreover, in $53 \%$ of cases, the left side would be expected to be perceptibly more well pneumatized, while $47 \%$ would be either symmetric or more well pneumatized on the right.

This study has several limitations. First, numerous conceivable paranasal sinus variants were not included for analysis, which might influence the interpretation of the number of variants when comparing the different groups in this study. The International Frontal Sinus Anatomy Classification (IFAC) system was introduced shortly after the APPS score ${ }^{32}$ and was subsequently validated for rater reliability. ${ }^{33}$ Therefore, variants described in the IFAC system are not included in the present study. Nevertheless, the variants tracked in the APPS score can predict the total sinus volume ${ }^{18}$ and have also been validated for interrater and intrarater reliability. ${ }^{17}$ The features of the APPS instrument allow for the simultaneous tracking of individual anatomic variations in addition to a global assessment of paranasal sinus pneumatization. Second, the cross-sectional design of this study limits comment on changes in sinus anatomy over time. These changes may even occur in nondiseased patients, and if so, would influence interpretation of anatomic findings in the setting of sinus disease. As mentioned previously, the male group was statistically younger than the female group which may confound these results, and the small number of Latinx/Hispanic patients in this study may limit generalizable conclusions about ethnicity as a factor in sinus pneumatization.

\section{CONCLUSION}

Patients without sinus disease have considerable variation in paranasal sinus anatomy. CT remains the standard for the radiographic evaluation of sinus disease. Our evaluation of sinus CTs using a validated metric revealed pervasive differences in overall sinus pneumatization between males and females and according to laterality, with the probability of encountering perceptible asymmetry approaching $70 \%$. We did not detect differences across ethnic groups or according to age. Improved understanding of baseline anatomic paranasal sinus variation may facilitate standardized assessment of sinus CT and aid clinician anatomical interpretation.

\section{ACKNOWLEDGMENTS}

The authors have no financial or proprietary interest in the subject matter of this article.

\section{REFERENCES}

1. Chang EH, Pezzulo AA, Meyerholz DK, et al. Sinus hypoplasia precedes sinus infection in a porcine model of cystic fibrosis. Laryngoscope. 2012 Sep;122(9):1898-1905. doi: 10.1002/lary.23392.

2. Eggesbø HB, Søvik S, Dolvik S, Eiklid K, Kolmannskog F. Proposal of a CT scoring system of the paranasal sinuses in diagnosing cystic fibrosis. Eur Radiol. 2003 Jun;13(6):1451-1460. doi: 10.1007/s00330-003-1825-8.

3. Seifert CM, Harvey RJ, Matthews JW, et al. Temporal bone pneumatization and its relationship to paranasal sinus development in cystic fibrosis. Rhinology. 2010 Jun;48(2):233-238.

4. Woodworth BA, Ahn C, Flume PA, Schlosser RJ. The delta F508 mutation in cystic fibrosis and impact on sinus development. 
Am J Rhinol. 2007 Jan-Feb;21(1):122-127. doi: 10.2500/ajr.2007.21.2905.

5. Pifferi M, Bush A, Caramella D, et al. Agenesis of paranasal sinuses and nasal nitric oxide in primary ciliary dyskinesia. Eur Respir J. 2011 Mar;37(3):566-571. doi: 10.1183/09031936.00068810.

6. Cho SH, Kim TH, Kim KR, et al. Factors for maxillary sinus volume and craniofacial anatomical features in adults with chronic rhinosinusitis. Arch Otolaryngol Head Neck Surg. 2010 Jun;136(6):610-615. doi: 10.1001/archoto.2010.75.

7. Kim HY, Kim MB, Dhong HJ, et al. Changes of maxillary sinus volume and bony thickness of the paranasal sinuses in longstanding pediatric chronic rhinosinusitis. Int J Pediatr Otorhinolaryngol. 2008 Jan;72(1):103-108. doi: 10.1016/j.jporl.2007.09.018.

8. Meyer TK, Kocak M, Smith MM, Smith TL. Coronal computed tomography analysis of frontal cells. Am J Rhinol. 2003 May-Jun:17(3):163-168.

9. Kim HJ, Friedman EM, Sulek M, Duncan NO, McCluggage C. Paranasal sinus development in chronic sinusitis, cystic fibrosis, and normal comparison population: a computed tomography correlation study. Am J Rhinol. 1997 Jul-Aug;11(4):275-281. doi: 10.2500/105065897781446676.

10. King VV. Upper respiratory disease, sinusitis, and polyposis. Clin Rev Allergy. 1991 Spring-Summer;9(1-2):143-157.

11. Prabhu AV, Branstetter BF 4th. The CT prevalence of arrested pneumatization of the sphenoid sinus in patients with sickle cell disease. AJNR Am J Neuroradiol. 2016 Oct;37(10):1916-1919. doi: 10.3174/ajnr.A4801.

12. Yonetsu K, Watanabe M, Nakamura T. Age-related expansion and reduction in aeration of the sphenoid sinus: volume assessment by helical CT scanning. AJNR Am J Neuroradiol. 2000 Jan;21(1):179-182.

13. Bolger WE, Butzin CA, Parsons DS. Paranasal sinus bony anatomic variations and mucosal abnormalities: CT analysis for endoscopic sinus surgery. Laryngoscope. 1991 Jan;101(1 Pt 1):56-64. doi: 10.1288/00005537-199101000-00010.

14. Dasar U, Gokce E. Evaluation of variations in the sinonasal region with computed tomography. World J Radiol. 2016 Jan 28;8(1):98-108. doi: 10.4329/wjr.v8.i1.98.

15. Earwaker J. Anatomic variants in sinonasal CT. Radiographics. 1993 Mar;13(2):381-415. doi: 10.1148/radiographics.13.2.8460226.

16. Lund VJ, Stammberger H, Fokkens WJ, et al. European position paper on the anatomical terminology of the internal nose and paranasal sinuses. Rhinol Suppl. 2014 Mar;24:1-34.

17. Marino MJ, Weinstein JE, Riley CA, Levy JM, Emerson NA, McCoul ED. Assessment of pneumatization of the paranasal sinuses: a comprehensive and validated metric. Int Forum Allergy Rhinol. 2016 Apr;6(4):429-436. doi: 10.1002/alr.21671.

18. Marino MJ, Riley CA, Kessler RH, McCoul ED. Clinician assessment of paranasal sinus pneumatization is correlated with total sinus volume. Int Forum Allergy Rhinol. 2016 Oct;6(10):1088-1093. doi: 10.1002/alr.21779.

19. Oluwole M, Russell N, Tan L, Gardiner Q, White P. A comparison of computerized tomographic staging systems in chronic sinusitis. Clin Otolaryngol Allied Sci. 1996 Feb;21(1):91-95.
20. Shah RK, Dhingra JK, Carter BL, Rebeiz EE. Paranasal sinus development: a radiographic study. Laryngoscope. 2003 Feb;113(2):205-209. doi: 10.1097/00005537-200302000-00002.

21. McGraw KO, Wong SP. A common language effect-size statistic. Psychol Bull. 1992;111(2):361-365. doi: 10.1037/0033-2909.111.2.361.

22. Lawson W, Patel ZM, Lin FY. The development and pathologic processes that influence maxillary sinus pneumatization. Anat Rec (Hoboken). 2008 Nov;291(11):1554-1563. doi: 10.1002/ar.20774.

23. Jun BC, Song SW, Park CS, Lee DH, Cho KJ, Cho JH. The analysis of maxillary sinus aeration according to aging process: volume assessment by 3-dimensional reconstruction by high-resolutional CT scanning. Otolaryngol Head Neck Surg. 2005 Mar;132(3):429-434. doi: 10.1016/j.otohns.2004.11.012.

24. Michel J, Paganelli A, Varoquaux A, et al. Determination of sex: interest of frontal sinus 3D reconstructions. J Forensic Sci. 2015 Mar;60(2):269-273. doi: 10.1111/1556-4029.12630.

25. Mohlhenrich SC, Heussen N, Peters F, Steiner T, Hölzle F, Modabber $A$. Is the maxillary sinus suitable in sex determination? A three-dimensional analysis of maxillary sinus volume and surface depending on sex and dentition. $J$ Craniofac Surg. 2015 Nov;26(8):e723-726. doi: $10.1097 /$ SCS.0000000000002226.

26. Prabhat M, Rai S, Kaur M, Prabhat K, Bhatnagar P, Panjwani S. Computed tomography based forensic gender determination by measuring the size and volume of the maxillary sinuses. $J$ Forensic Dent Sci. 2016 Jan-Apr;8(1):40-46. doi: 10.4103/0975-1475.176950.

27. Emirzeoglu M, Sahin B, Bilgic S, Celebi M, Uzun A. Volumetric evaluation of the paranasal sinuses in normal subjects using computer tomography images: a stereological study. Auris Nasus Larynx. 2007 Jun;34(2):191-195. doi: 10.1016/j.anl.2006.09.003.

28. Badia L, Lund VJ, Wei W, Ho WK. Ethnic variation in sinonasal anatomy on CT-scanning. Rhinology. 2005 Sep;43(3):210-214.

29. Soler ZM, Mace JC, Litvack JR, Smith TL. Chronic rhinosinusitis, race, and ethnicity. Am J Rhinol Allergy. 2012

Mar-Apr;26(2):1 10-116. doi: 10.2500/ajra.2012.26.3741.

30. Fernandes CL. Forensic ethnic identification of crania: the role of the maxillary sinus-a new approach. Am J Forensic Med Pathol. 2004 Dec;25(4):302-313. doi: 10.1097/01.paf.0000146379.85804.da.

31. Keast A, Yelavich S, Dawes $P$, Lyons B. Anatomical variations of the paranasal sinuses in Polynesian and New Zealand European computed tomography scans. Otolaryngol Head Neck Surg. 2008 Aug;139(2):216-221. doi: 10.1016/j.otohns.2008.05.014.

32. Wormald PJ, Hoseman W, Callejas C, et al. The International Frontal Sinus Anatomy Classification (IFAC) and classification of the Extent of Endoscopic Frontal Sinus Surgery (EFSS). Int Forum Allergy Rhinol. 2016 Jul;6(7):677-696. doi: 10.1002/alr.21738.

33. Villarreal R, Wrobel BB, Macias-Valle LF, et al. International assessment of inter- and intrarater reliability of the International Frontal Sinus Anatomy Classification system. Int Forum Allergy Rhinol. 2019 Jan;9(1):39-45. doi: 10.1002/alr.22200.

This article meets the Accreditation Council for Graduate Medical Education and the American Board of Medical Specialties Maintenance of Certification competencies for Patient Care and Medical Knowledge.

(C2020 by the author(s); licensee Ochsner Journal, Ochsner Clinic Foundation, New Orleans, LA. This article is an open access article distributed under the terms and conditions of the Creative Commons Attribution (CC BY) license (creativecommons.org/licenses/by/4.0/legalcode) that permits unrestricted use, distribution, and reproduction in any medium, provided the original author(s) and source are credited. 